\title{
Teleconsultation for the Pharmaceutical Care of HIV Outpatients in Receipt of Home Antiretrovirals Delivery: Clinical, Economic, and Patient-Perceived Quality Analysis
}

\author{
Luis Margusino-Framiñán ${ }^{12}$, Purificación Cid-Silva ${ }^{12}$, Ángeles Castro- \\ Iglesias $^{23}$, Álvaro Mena-de-Cea ${ }^{23}$, Iria Rodríguez-Osorio ${ }^{23}$, Berta Pernas- \\ Souto $^{23}$, Pilar Vázquez-Rodríguez ${ }^{23}$, Soledad López-Calvo ${ }^{23}$, Isabel Martín- \\ Herranz ${ }^{1}$
}

${ }^{1}$ Pharmacy Service, A Coruña University Hospital, A Coruña, Spain.

${ }^{2}$ Division of Clinical Virology, A Coruña Biomedical Research Institute (INIBIC), A Coruña University Hospital, SERGAS, A Coruña University (UDC), A Coruña, Spain.

${ }^{3}$ Infectious Diseases Unit, Internal Medicine Service, A Coruña University Hospital, A Coruña, Spain.

\begin{abstract}
Background/Introduction: Pharmacist teleconsultations, combined with home drug delivery or mail-order pharmacy (MOP), can help hospital outpatients with difficulties accessing treatment. The objectives of this study are to describe a teleconsultation protocol and to evaluate clinical, economic, and patient-perceived quality results.

Materials and Methods: A cohort observational study was carried out for 3 years on HIV outpatients. Clinical variables were adherence, plasma HIV-RNA, and CD4+ levels. A pharmacoeconomic analysis was carried out through a cost-minimization study. Patient-perceived quality was assessed through a satisfaction survey. Simple random sampling was performed for 95\% safety, accuracy $\pm 1 \%$, and losses $\pm 20 \%$.

Results: The 38 participants (sample size) consisted of $82 \%$ male patients, aged $44.7 \pm 8.4$ years. There were 854 teleconsultations and $100 \%$ treatment adherence. All HIV outpatients kept virally suppressed $(\mathrm{p}=1.00)$ and maintained a controlled immunological level $(\mathrm{p}=0.87)$. The economic evaluation revealed $137 \pm 23 €$ patient/year costs-saved and $18.5 \pm 7.2 \mathrm{~h} /$ patient/year working time gained. Patient-perceived quality average score was $>9.4$ out of 10 in all items; the most valued factors were the saving of direct costs and reconciliation with work commitments $(45 \%)$ and the least valued attributes were making the payment for the shipment and having to adjust to a telephone appointment (41\%).

Discussion/Conclusions: A teleconsultation protocol associated with home antiretrovirals delivery or MOP obtains a high degree of satisfaction from the HIV hospital outpatients receiving treatment, without repercussions on the therapeutic objectives and with the saving of important direct costs for the patient and indirect costs in relation to labor productivity.
\end{abstract}

Keywords: Teleconsultation, telepharmacy, HIV, antiretroviral agents, home drug delivery, mail-order pharmacy, pharmaceutical care, telemedicine 


\section{Introduction}

The World Health Organization (WHO) defines telemedicine as the provision of health services, where distance is a critical factor, by any health professional through the use of information and communication technologies for the exchange of valid information for diagnosis, treatment and prevention of diseases and injuries, research and evaluation, and the permanent education of healthcare providers, all in the interest of improving the health of individuals and their communities. ${ }^{1}$ Telepharmacy is, therefore, to be considered a part of telemedicine and has been defined as a method used in pharmaceutical practice in which a pharmacist uses communication technologies to provide patient care services or to supervise pharmacy activities that may include, but are not limited to, the validation and monitoring of treatments, dispensing, the validation of the preparation of medications, providing information and advice to patients, clinical consultations, evaluation of results, support for decisionmaking, and providing information on medications. ${ }^{2}$

In healthcare systems around the world, home drug delivery (HDD) in Europe, called mail-order pharmacy (MOP) in the United States of America, has been used alongside telepharmacy for the provision of pharmacological treatments at home or the workplace. Both HDD and MOP facilitate better access to medication, especially in rural regions or those involving great geographical dispersion, and those that have other difficulties of access in relation to pharmaceutical care, but differ mainly in that MOP is performed from community pharmacies and HDD from hospital pharmacy services (HPSs) for certain medications. HDD has produced very good clinical results in terms of both its level of efficiency and its therapeutic safety ${ }^{3-7}$; also, MOP has showed good clinical results in terms of adherence to treatment in chronic diseases, mainly in diabetes. ${ }^{8-13}$

In addition to improving accessibility to treatment, HDD/ MOP prevents the situation whereby the patient has to travel periodically to the community pharmacy or the hospital pharmacy, and this promotes work and family reconciliation, decreases direct and indirect costs both from the patient's and from the social perspective and increases overall satisfaction with the health system that provides the service. These aspects are necessary to include in the evaluation of this healthcare procedure; however, they have been evaluated in very few studies ${ }^{14-16}$ or have been analyzed from the perspective of publics or private pharmacy benefit management plans (Medicare Part D, Medicaid, U.S. Department of Defense Healthcare Plan, Retirement Systems, etc.). ${ }^{17-20}$

There are no published studies that evaluate any of these aspects in Spain, where pharmaceutical care $(\mathrm{PhC})$ for outpatients using HPSs is required for medicines classified as "hospital diagnosisHD' or "hospital use-H." These medications cannot be dispensed at community pharmacies and should only be dispensed at hospital pharmacy, with a monthly or bimonthly periodicity. Current regional regulations establish, for community pharmacies, that HDD is authorized for, among other patients, stable chronically ill outpatients, in special circumstances, with a guaranteed medical prescription and with prior dispensing of that same medication; so HDD from HPS could be applied to outpatients treated through pharmaceutical telecare with HD-medicines or $\mathrm{H}$ medicines.

In our hospital, pharmaceutical care to outpatients with HD-medicines or H-medicines is provided by pharmacists specialized in pathologies or specific therapeutic areas, in monographic consultations with a very high degree of outpatient satisfaction. ${ }^{21}$ The health area covered by our hospital has a highly dispersed population (550,000 inhabitants, largely rural, aging, and with limited access by public or private means of transport from certain remote locations). The pharmacists responsible for pharmaceutical care to outpatients in our hospital, considering this, assess patients' suitability for HDD and pharmaceutical telecare, and promote telepharmacy within the strategic plan of the Pharmacy Service. To this end, they have approved and implemented a telepharmacy protocol based on teleconsultation in relation to pharmaceutical care ( $\mathrm{TcPhC})$ with HDD for clinically stable outpatients with difficulties accessing treatment for various reasons (labor, economic, family, etc.). These patients may currently include solid organ transplant patients, HIV patients, patients with arthropathies, and patients with pulmonary or neurological 
diseases. It is interesting, therefore, to investigate what repercussions $\mathrm{TcPhC}-\mathrm{HDD}$ has at various levels, so that, according to the results of this examination, health managers can make decisions based on evidence regarding this aspect of telepharmacy. More specifically, in the field of persons living with HIV on antiretroviral therapy, a recent survey of pharmacists working in different settings revealed the need of more research about the impact of MOP on adherence or health outcomes, the skills to overcome the lack of face-toface consultations, or when to switch patients to HDD or MOP. ${ }^{22}$

In accordance with that mentioned, the objectives of this study are to describe HIV patient candidates for the $\mathrm{TcPhC}-\mathrm{HDD}$ protocol, the implementation phases required, and the care circuit and subsequently to evaluate the clinical, economic, and patient-perceived quality results postimplementation.

\section{Materials and Methods}

\section{TCPHC-HDD protocol}

The TcPhC-HDD protocol for HIV outpatients was coordinated between the hospital pharmacists and the physicians responsible for the Hospital Immunodeficiency Unit (HIU) and the inclusion and exclusion criteria for the candidate patients were established. The inclusion criteria were as follows: adult HIV outpatients receiving antiretroviral treatment (ART); at least 6 months of follow-up in the HIU and HPS before inclusion in the protocol; stable patients with chronic controlled infection objectified by two negative viral loads in consecutive determinations during at least the past 6 months; and 100\% adherence to ART (according to medical and pharmaceutical criteria registered in the course of the electronic clinical medical record). Exclusion criteria were as follows: patients with ART change motivated by virological failure or the development of adverse effects (until resolution of the reason for change); breach of a previous appointment during the last year in outpatient hospital pharmacy or medical clinic without scheduling a replacement; concomitant treatment with other HD-medicines or $\mathrm{H}$-medicines that require face-to-face consultation in HPS; and appointments scheduled at the hospital during the dispensing period allowed by the "single clinical act" (administrative procedure of scheduling several appointments in different outpatient hospital clinics on the same day).

Subsequently, in collaboration with the hospital agents involved (pharmacist, physicians, the Pharmacy Service Manager, and the Hospital Manager and Hospital Medical Director), the document "TcPhC-HDD Work Instruction", was drafted and included within the quality certification program of the HPS, the Patient Request and Informed Consent Form was designed to facilitate the patients' access to this protocol and submitted for the approval of the Hospital Medical Director. The document "TcPhC-HDD Work Instruction"' established the following assistance circuit:

(1) Patient inclusion: pharmacist gives a detailed explanation to the patient of the $\mathrm{TcPhC}-$ HDD protocol and, if the patient agrees, she/he signs the "Patient Request and Informed Consent Form.",

(2) Patient assignment to the Teleconsultation (2.2FT) provision in the agenda of the monographic consultation for the pharmaceutical care of HIV patients.

(3) Bi-monthly telematic consultation after appointment and the conducting of clinical interviews according to the same standard procedure for pharmaceutical care, which is in effect for face-to-face consultations (mainly treatment assessment, clinical variables monitoring, adherence evaluation, pharmacological interactions, and adverse effects monitoring). Since hospital extranet does not absolutely guarantee the privacy/confidentiality of the communication with outpatients, they receive telematic 
consultation by telephone call wherever they are (home, workplace, etc.). Therefore, no special technical requirements are needed to engage in the program.

(4) Documentation of the clinical interviews and the pharmaceutical care provided in the course of the clinical pharmacy activity, as indicated by the electronic medical record.

(5) Electronic account of the quantity of dispensed units from the pharmacy service drug stock.

(6) Preparation and packing of medication by the pharmacy technician of the HPS.

(7) Coordination of HDD with the distribution company as arranged by the hospital through the HPS administrator.

(8) Home reception of the medicine within $24 \mathrm{~h}$ and patient payment of transportation costs.

\section{Study design}

This consisted of a pre-post cohort observational retrospective study on interventions carried out on patients included in the TcPhC-HDD protocol at some time from July 2014 to July 2017. The specific intervention looked at by this study is patient inclusion in the TcPhC-HDD protocol in any of the face-to-face pharmaceutical care consultations carried out with the patient during the study period.

Three types of variables were evaluated: clinical, pharmacoeconomic, and in relation to patientperceived quality. Three clinical variables were included: ART patient adherence, plasma HIVRNA, and CD4+ levels. Two pharmacoeconomic variables were included: (1) direct costs avoided from the patient's perspective (cost-minimization study assuming the same final clinical resultscosts of public or private transportation that the patient would have had to incur to go to the HPS consultation less the costs actually paid by the patient to the company responsible for drug home delivery) and (2) indirect costs (lost work hours avoided by the avoidance of the need to travel the hospital in active patients). Finally, patient-perceived quality was assessed through eight variables extracted from each household and an anonymous Teleconsultation satisfaction survey, which was based on the outpatient satisfaction survey regarding the HPS quality certification system (Fig. 1). 
- general data:

- Age (years): Gender:

- Employment situation (employee, unemployed, pensioner).

- Means of transport to travel to a clinic in the HPS.

- Who pays the expenses of this trip to the hospital?

- How long does it take you to go to your appointment with the HPS?

- How did you know the TcPhC-HDD?:

- Pharmacist proposal.

- Nurse/Physician proposal.

- Patient's request.

- Other patients.

- Who do you think has established this protocol?:

- Regional Health Service.

- Hospital authorities.

- Hospital Pharmacy Service.

- SURVEY (Indicate from 0 to 10 your degree of satisfaction with TcPhC-HDD (0 the worst; 10 the best).

\begin{tabular}{|l|c|c|c|c|c|c|c|c|c||}
\hline Privacy and confidentiality & 0 & 1 & 2 & 3 & 4 & 5 & 6 & 7 & 8 \\
\hline Courier service & 0 & 1 & 2 & 3 & 4 & 5 & 6 & 7 & 8 \\
\hline Pharmacist's phone call appointment & 0 & 1 & 2 & 3 & 4 & 5 & 6 & 7 & 8 \\
\hline Phone call understanding & 0 & 1 & 2 & 3 & 4 & 5 & 6 & 7 & 8 \\
\hline Drug information service provided & 0 & 1 & 2 & 3 & 4 & 5 & 6 & 7 & 8 \\
\hline Treatment and kindness & 0 & 1 & 2 & 3 & 4 & 5 & 6 & 7 & 8 \\
\hline Teleconsultation duration & 0 & 1 & 2 & 3 & 4 & 5 & 6 & 7 & 8 \\
\hline Overall rating & 0 & 1 & 2 & 3 & 4 & 5 & 6 & 7 & 8 \\
\hline
\end{tabular}

\section{- finAl questions:}

- What do you value most about TCPhC-HDD? (note only one option):
- Savings on travel expenses.
- Family reconciliation.
- Labor reconciliation.
- Improvement in privacy and confidentiality.
- Others (indicate).

- What is the least value of TcPhC-HDD? (note only one option):

- Having to pay transportation expenses.

- Telephone communication.

- Lack of privacy that supposes that a carrier makes the delivery.

- Have to be alert on the day of the pharmacist's call.

- Others (indicate).

- Would you prefer that all consultations be with physical presence at the HPS and exit the TcPhC-HDD?:
- Yes.

- No.

Fig. 1. Teleconsultation pharmaceutical care-HDD satisfaction survey. HPS, Hospital Pharmacy Service; $\mathrm{TcPhC}-\mathrm{HDD}$, teleconsultation of pharmaceutical care with home drug delivery 


\section{Statistical analysis}

Simple random sampling was performed among patients involved in the TcPhC-HDD protocol for $95.0 \%$ safety, accuracy $\pm 1.0 \%$, and losses $\pm 20.0 \%$. Quantitative variables are expressed here as means \pm standard deviation and qualitative variables as percentages with a confidence interval of 95\%. Statistical significance was calculated with the Fisher exact test and the Mann-Whitney test. Results were considered statistically significant when their $p$-value was $<0.05$. Statistical analysis was carried out using the Epidat 3.1 program.

\section{Ethics}

Access to the patient's clinical history data was based on "Patropresvih Study," as classified by the National Health Authorities in 2014 as a "Post-authorization study with other aims than the prospective follow-up" and approved by the Regional Clinical Research Ethics Committee. Previously, patients had already signed a consent to participate in the TcPhC-HDD protocol, and had given verbal consent to participation in this study.

\section{Results}

Of the 1,119 HIV patients on ART at our institution, 81 patients were selected for inclusion in the TcPhC-HDD protocol for 3 years, during which 354 face-to-face consultations and 854 teleconsultations associated with home drug deliveries were carried out. Of these 81 patients, 38 constituted the sample analyzed according to the statistical design applied. All patients had a 100\% adherence to treatment. Table 1 shows the main characteristics of the study population.

Table 1. Characteristics of the Study Population

$\begin{array}{ll}\text { Demographic characteristics } & \\ \text { Age, years (mean } \pm \text { SD) } & 44.7 \pm 8.4 \\ \text { Gender, men } & 81.6 \% \\ \text { TcPhC-HDD-related variables } & \\ \text { Employment situation } & 78.9 \% \\ \quad \text { Labor-active patients } & 13.2 \% \\ \quad \text { Unemployed } & 7.9 \% \\ \quad \text { Pensioner } & \\ \text { Means of transport to HPS } & 78.2 \% \\ \quad \text { Private (car) } & 21.8 \% \\ \text { Public (bus or train) } & \\ \text { Patient transport payment } & 93.3 \% \\ \quad \text { Self-payment } & 6.7 \% \\ \text { Public aids } & 145.8 \pm 122.2 \\ \text { Time to arrive and return to face-to-face HPS appointment from } \\ \text { home or workplace, minutes (mean } \pm \text { SD) } & \\ \text { Way of TcPhC-HDD knowledge } & 60.5 \% \\ \text { Pharmacist proposal } & 21.1 \% \\ \text { Patient's request } & 13.2 \% \\ \quad \text { Nurse/physician proposal } & 5.2 \% \\ \text { Other patients } & \\ \text { Patient's beliefs about who has established TcPhC-HDD } & 57.9 \% \\ \quad \text { Doesn't know } & 26.3 \% \\ \text { Hospital pharmacy service } & 10.5 \% \\ \text { Hospital authorities } & \end{array}$


Regional health service

Best valued factor of $\mathrm{TcPhC} \pm \mathrm{HDD}$ (multiple answer)

Savings on travel expenses $\quad 45.8 \%$

Labor reconciliation $\quad 45.8 \%$

Family reconciliation $\quad 31.6 \%$

Improvement in privacy and confidentiality $\quad 21.5 \%$

Others $\quad 5.3 \%$

Worst factor of TcPhC-HDD (multiple answer)

$\begin{array}{ll}\text { Having to pay transportation expenses } & 41.7 \% \\ \text { Prior appointment of the telephone call } & 41.0 \%\end{array}$

Telephone communication $\quad 2.6 \%$

Lack of privacy in deliveries $\quad 2.6 \%$

Others $0 \%$

Preference of face-to-face consultations and exit TcPhC-HDD
$\quad$ No

Yes $\quad 0 \%$

Time on TcPhC-HDD protocol, months (median) 19.9

Loss of follow-up $0 \%$

HPS, hospital pharmacy service; SD, standard deviation; TcPhC \pm HDD, teleconsultation of pharmaceutical care with home drug delivery

At the clinical level, ART effectiveness variables showed that $100 \%$ of the patients maintained an HIV viral load of undetectable $(p=1.00)$ and an immunological level $(\mathrm{CD} 4+=658 \pm 224 / \mu \mathrm{L} ; p=$ 0.87 ), after an average of 1 year of the pharmaceutical follow-up protocol. The economic evaluation of the variables related to direct and indirect costs revealed that the patient/year costssaved were $137 \pm 23 €$ and the patient/year working time gained was $18.5 \pm 7.2 \mathrm{~h}$.

The satisfaction survey indicated that the factors most valued by the outpatients were the saving of direct costs $(45.8 \%, 95 \%$ CI $30.7-61.0 \%)$ and labor time recovery $(45.8 \%, 95 \%$ CI $30.7-61.0 \%)$ and the least valued factors were having to make the payment for the shipment $(41.7 \%, 95 \%$ CI 26.7-56.6\%) and having to adjust to a telephone appointment (41\%). Figure 2 shows the results for the eight items included in the satisfaction survey. 


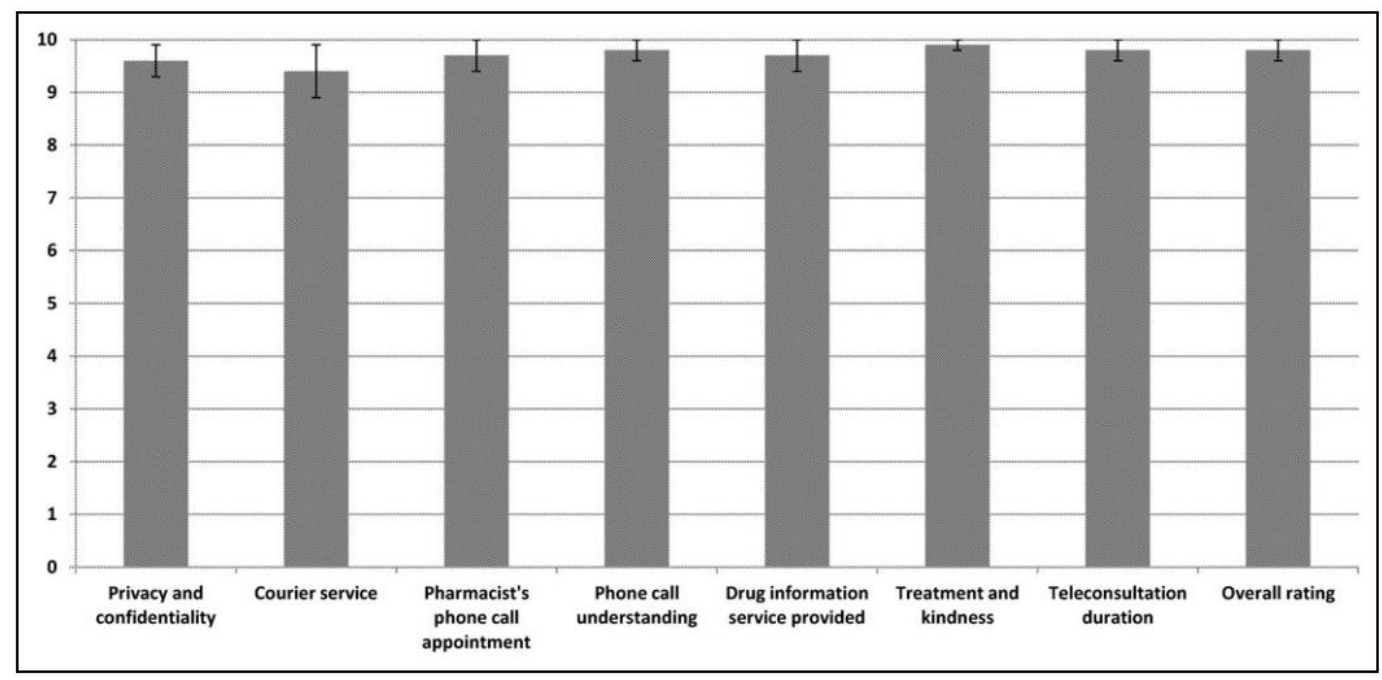

Fig. 2. Results of teleconsultation of pharmaceutical care-HDD satisfaction survey.

\section{Discussion}

The introduction of telepharmacy as a line of action for health services allows patients to have access to pharmaceutical healthcare services remotely through communication technologies with a wide range of services included, ranging from validation or pharmaceutical monitoring, information about medicines, health education, and medication reconciliation services to home delivery. ${ }^{23,24}$ Within this process, the TcPhC-HDD pharmacy protocol proposed in our HPS aimed to allow access to pharmaceutical care for HIV patients with access difficulties as regards the hospital, either because of work, family, economic, or geographical dispersion reasons. This was implemented to maintain the therapeutic goal of ART, that is, the negativization of HIV load and the level of immunocompetence achieved. Assessing the baseline characteristics of the patients included in the study (young adult labor-active population using self-payment private means of transport for their access to the HPS) and the best valued items shown by the patients in the satisfaction survey (saving on travel expenses and labor/family reconciliation), we consider that stable HIV patient is an excellent target population since it meets the criteria that justify the implementation of this telecare protocol.

At a clinical level, the results of our study have shown that the TcPhC-HDD protocol has not caused statistically or clinically significant decreases in CD4+ lymphocyte levels, and, in addition, it has maintained virally suppressed all patients included in the study. This is an essential point for the positive evaluation of the program, since the value of HIVRNA is the main variable of effectiveness of ART. ${ }^{25}$ Very few clinical studies have evaluated the impact of telepharmacy on patients monitored through these technologies. Some recent studies have demonstrated the validity of this type of pharmaceutical care on asthma, diabetes, and patients at hospital discharge. ${ }^{26-30}$ However, very few studies have analyzed the influence of telepharmacy on the clinical outcome of HIV patients receiving ART or the evaluation has been limited to the influence of MOP for improving medication adherence to ART. ${ }^{31}$ The first study that evaluated the impact of telepharmacy for HIV patients receiving ART was carried out in Spain with regard to the "HospitalVirtual" experience of the hospital clinic of Barcelona; this was encompassed within a telemedicine computer platform. It was demonstrated through a randomized clinical trial that telemedicine is a safe tool for home care of chronic HIV patients and should be considered as an appropriate support service for the management of chronic HIV infection. ${ }^{32-34}$ More recently, 
Castellino et al. ${ }^{35}$ showed that home delivery did not generate negative results in terms of adherence to ART by HIV patients, nor on viral load of HIV or on CD4+ lymphocytes; this is in line with our study, which seems to corroborate these previous results of the Barcelona group.

Another important issue related to telepharmacy is the promotion of the accessibility of pharmaceutical care and pharmacological treatments to outpatients. This is especially, although not exclusively, significant where patients live in rural populations, in very dispersed population areas or with difficulties in accessing face-to-face healthcare from their areas of residence or work. Based on this idea, a potential disparity between rural and urban patients' access to clinical pharmacy services has been identified and telepharmacy could decrease this disparity. ${ }^{36,37}$ In fact, some studies have shown the benefits of telepharmacy at this level. ${ }^{38,39}$ In line with these results, our study has revealed that the average time spent attending the HPS outpatient clinic is almost 2.5 hours, which confirms the enormous distance and difficulties in accessing for HIV outpatients from home or workplace. In addition, one of the aspects most valued by HIV outpatients included in $\mathrm{TcPhC}-\mathrm{HDD}$ is precisely the work-life reconciliation, especially considering that $80 \%$ of them are labor active.

The third aspect to consider is the economic one. No study has previously evaluated the economic impact of HDD or MOP from the HIV outpatient perspective; most of the published studies in other chronic pathologies have been carried out from the perspective of the service provider or payer and suggest that these drug-dispensing systems do not provide a clear economic benefit ${ }^{11,40}$ or result in a marginal opportunity cost. ${ }^{8,41-44}$ Conversely, our study results show that the travel every month or every 2 months to the HPS for face-to-face consultation and drug dispensing represents an important expense that the patient assumes and is the cause of a loss of relevant productivity throughout the year and demonstrate a clear benefit at this level; unfortunately, our results cannot be compared with those of other studies, since these exact parameters have not been previously analyzed.

Finally, it is worth highlighting that in accordance with other research studies, ${ }^{6}$ excellent results were obtained through the anonymous survey of hospital outpatient satisfaction in the protocol; this showed a high perceived quality (with an overall result of 9.7 points out of 10), both at the organizational level and in terms of the functioning of the system (items 1-4) and at the pharmaceutical care level (items 5-7). However, some aspects of the protocol could be improved, as stated by the patients, in relation to telephone call appointment and delivery payments. In addition, none of the surveyed patients felt they would prefer to abandon the TcPhC-HDD protocol and return to prior types of consultations. In our opinion, this high satisfaction rate is motivated because our protocol hospital includes not only the periodic sending of medication but also the maintenance of teleconsultation of pharmaceutical care, as patients (HIV or not) have claimed in different studies. ${ }^{45,46}$

From our point of view, the main limitation of this study (and, therefore, of the extrapolation of results to the entire population of HIV outpatients treated by the pharmacy service of our hospital) is that the TcPhC-HDD protocol currently includes HIV patients who have previously shown be adherent to treatment and clinically controlled. This could have led to a results bias. However, we considered it necessary to explore first using this type of stable patient whether or not the protocol causes negative results with regard to their clinical situation. Thus, in later phases of implementation (and given the excellent results obtained), we can extend our researches to other HIV patients and assess in a further study whether, as a result of better access to treatment, other patients could actually improve their clinical results. In contrast, our study has clear strengths taking into account the HIV outpatient perspective, since it brings new findings not previously published in relation to the economic benefit of pharmacist teleconsultation with HDD/MOP and, above all, excellent results are presented for the first time on the clinical variables that define the progression of HIV disease and patient survival. 
In summary, $\mathrm{TcPhC}-\mathrm{HDD}$ obtains a high degree of satisfaction from HIV hospital outpatients receiving ART and has no negative repercussion on the therapeutic objectives in terms of HIV plasma viral load or CD4+ lymphocytes plasma level, and in addition saves on direct costs, which are important for the patient and on indirect costs with regard to labor productivity. We consider that we are in a position to expand the inclusion criteria for HIV patients and analyze the results then produced.

\section{Authors' Contributions}

All authors declare that they made substantial contribution to the concept or design of the work, or to acquisition, analysis, or interpretation of data; drafted the article or revised it critically for important intellectual content; approved the version to be published; and each author should have participated sufficiently in the work to take public responsibility for appropriate portions of the content.

\section{Ethical Statement}

Access to the patient's clinical history data is included in the "Patropresvih Study," approved by the Clinical Research Ethics Committee of the local health authority. Patients have given written consent to participation in the study and verbal consent to participation in the protocol.

\section{Disclosure Statement}

No competing financial interests exist.

\section{REFERENCES}

1. World Health Organization. Telemedicine. Opportunities and developments in members states. 2010. Available at www.who.int/goe/publications/goe_telemedicine_2010.pdf (last accessed December 30, 2017).

2. Alexander E, Butler CD, Darr A, Jenkins MT, Long RD, Shipman CJ, et al. ASHP statement on telepharmacy. Am J Health Syst Pharm 2017;74:e236-e241.

3. Rose JL. Improved and expanded pharmacy care in rural Alaska through telepharmacy and alternative methods demonstration project. Int J Circumpolar Health 2007;66 Suppl 1:14-22.

4. Inch J, Notman F, Watson M, Green D, Baird R, Ferguson J, et al. Tele-pharmacy in rural Scotland: A proof of concept study. Int J Pharm Pract 2017;25: 210-219.

5. Friesner DL, Scott DM, Rathke AM, Peterson CD, Anderson HC. Do remote community telepharmacies have higher medication error rates than traditional community pharmacies? Evidence from the North Dakota Telepharmacy Project. J Am Pharm Assoc 2011;51:580-590.

6. Clifton GD, Byer H, Heaton K, Haberman DJ, Gill H. Provision of pharmacy services to underserved populations via remote dispensing and two-way videoconferencing. Am J Health Syst Pharm 2003;60:2577-2582.

7. Skrei A, Rundquist MM. Pharmacy services in Telepharmacy: How's it working, where it's working, and what's required to practice in this new setting. Adv Pharm 2017;1:1-7.

8. Devine S, Vlahiotis A, Sundar H. A comparison of diabetes medication adherence and healthcare costs in patients using mail order pharmacy and retail pharmacy. J Med Econ 2010;13:203-211.

9. Duru OK, Schmittdiel JA, Dyer WT, Parker MM, Uratsu CS, Chan J, et al. Mailorder pharmacy use and adherence to diabetes-related medications. Am J Manag Care 2010;16:33-40.

10. Fernandez EV, McDaniel JA, Carroll NV. Examination of the link between medication adherence and use of mail-order pharmacies in chronic disease states. J Manag Care Spec Pharm 2016;22:1247-1259.

11. Martin C, Odell K, Cappelleri JC, Bancroft T, Halpern R, Sadosky A. Impact of a novel cost-saving pharmacy program on pregabalin use and health care costs. J Manag Care Spec Pharm 2016;22:132144.

12. Schmittdiel JA, Karter AJ, Dyer WT, Chan J, Duru OK. Safety and effectiveness of mail order pharmacy use in diabetes. Am J Manag Care 2013;19:882-887. 
13. Zhang L, Zakharyan A, Stockl KM, Harada ASM, Curtis BS, Solow BK. Mail-order pharmacy use and medication adherence among Medicare Part D beneficiaries with diabetes. J Med Econ 2011;14:562567.

14. Snoswell C, Smith AC, Scuffham PA, Whitty JA. Economic evaluation strategies in telehealth: Obtaining a more holistic valuation of telehealth interventions. J Telemed Telecare 2016;23:792-796.

15. Kooy MJ, Van Geffen ECG, Heerdink ER, Van Dijk L, Bouvy ML. Patients' general satisfaction with telephone counseling by pharmacists and effects on satisfaction with information and beliefs about medicines: Results from a cluster randomized trial. Patient Educ Couns 2015;98:797-804.

16. Garrelts JC, Gagnon M, Eisenberg C, Moerer J, Carrithers J. Impact of telepharmacy in a multihospital health system. Am J Health-Syst Pharm 2010;67:1456-1462.

17. Carroll NV. A comparison of costs of Medicare Part D prescriptions dispensed at retail and mail order pharmacies. J Manag Care Spec Pharm 2014;20:959-967.

18. Linton A, Garber M, Fagan NK, Peterson M. Factors associated with choice of pharmacy setting among DoD health care beneficiaries aged 65 years or older. J Manag Care Pharm 2007;13:677-686.

19. Valluri S, Seoane-Vazquez E, Rodriguez-Monguio R, Szeinbach SL. Drug utilization and cost in a Medicaid population: A simulation study of community vs. mail order pharmacy. BMC Health Serv Res 2007;7:122.

20. Visaria J, Seoane-Vazquez E, Szeinbach SL, Rodriguez-Monguio R. Comparing the cost of community pharmacy and mail-order pharmacy in a US retirement system. Int J Health Plann Manage 2012;27:e41-e50.

21. Margusino-Framiñán L, Cid-Silva P, Martínez-Roca C, Garcia-Queiruga M, Fernández-Gabriel E, Mateos-Salvador M, et al. Implementation of specialized pharmaceutical care hospital outpatient clinics in a hospital pharmacy department. Farm Hosp 2017;41:660-666.

22. Kibicho J, Owczarzak J, Pinkerton SD. Opinions of a small sample of pharmacists about pharmacy setting and patient adherence to antiretroviral therapy. J Manag Care Pharm 2012;18:446-452.

23. Tzanetakos G, Ullrich F, Meuller K. Telepharmacy rules and statutes: A 50-state survey. Rural Policy Brief 2017;4:1-4.

24. Keeys C, Kalejaiye B, Skinner M, Eimen M, Neufer J, Sidbury G, et al. Pharmacistmanaged inpatient discharge medication reconciliation: A combined onsite and telepharmacy model. Am J Health Syst Pharm 2014;71:2159-2166.

25. AIDS Study Group (GESIDA) of the Spanish Society of Infectious Diseases and Clinical Microbiology and the National AIDS Plan. Executive summary of the GESIDA/National AIDS plan consensus document on antiretroviral therapy in adults infected by the human immunodeficiency virus (updated January 2017). Enferm Infecc Microbiol Clin 2017; pii: S0213-005X(17)30131-3.

26. Rebello KE, Gosian J, Salow M, Sweeney P, Rudolph JL, Driver JA. The rural PILL program: A postdischarge telepharmacy intervention for rural veterans. J Rural Health 2017;33:332-339.

27. Brown W, Scott D, Friesner D, Schmitz T. Impact of telepharmacy services as a way to increase access to asthma care. J Asthma 2017;54:961-967.

28. Maxwell LG, McFarland MS, Baker JW, Cassidy RF. Evaluation of the impact of a pharmacist-led telehealth clinic on diabetes-related goals of therapy in a veteran population. Pharmacotherapy 2016;36:348-356.

29. Young HN, Havican SN, Griesbach S, Thorpe JM, Chewning BA, Sorkness CA. Patient And phaRmacist Telephonic Encounters (PARTE) in an underserved rural patient population with asthma: Results of a pilot study. Telemed J E Health 2012;18:427-433.

30. Bynum A, Hopkins D, Thomas A, Copeland N, Irwin C. The effect of telepharmacy counseling on metered-dose inhaler technique among adolescents with asthma in rural Arkansas. Telemed J E Health 2001;7:207-217.

31. Gross R, Zhang Y, Grossberg R. Medication refill logistics and refill adherence in HIV. Pharmacoepidemiol Drug Saf 2005; 14:789-793.

32. León A, Cáceres C, Fernández E, Chausa P, Martin M, Codina C, et al. A new multidisciplinary home care telemedicine system to monitor stable chronic human immunodeficiency virus-infected patients: A randomized study. PLoS One 2011;6:e14515.

33. Blanch Andreu J, Cáceres Taladriz C, Nomdedeu M, Rousaud Pares A, Gómez Aguilera EJ, García F. Telemedicine and HIV (in Spanish). UOC Pap 2007;4: 1-8.

34. Caceres C, Gomez EJ, Garcia F, Gatell JM, del Pozo F. An integral care telemedicine system for HIV/AIDS patients. Int J Med Inform 2006;75: 638-642.

35. Castellino S, Miah H, Auyeung V, Vogt F. Determination of the influence of home delivery of HIV therapy on virological outcomes and adherence. Int J STD AIDS 2015;26:93-97.

36. Goodridge D, Marciniuk D. Rural and remote care: Overcoming the challenges of distance. Chron Respir Dis 2016;13:192-203.

37. Patterson BJ, Kaboli PJ, Tubbs T, Alexander B, Lund BC. Rural access to clinical pharmacy services. $J$ Am Pharm Assoc 2014;54:518-525. 
38. Poulson LK, Nissen L, Coombes I. Pharmaceutical review using telemedicine-A before and after feasibility study. J Telemed Telecare 2010;16:95-99.

39. Rose JL. Improved and expanded pharmacy care in rural Alaska through telepharmacy and alternative methods demonstration project. Int J Circumpolar Health 2007;66 Suppl 1:14-22.

40. Khandelwal N, Duncan I, Rubinstein E, Ahmed T, Pegus C. Community pharmacy and mail order cost and utilization for 90-day maintenance medication prescriptions. J Manag Care Pharm 2012;18:247255.

41. Johnsrud M, Lawson KA, Shepherd MD. Comparison of mail-order with community pharmacy in plan sponsor cost and member cost in two large pharmacy benefit plans. J Manag Care Pharm 2007;13:122134.

42. Adams E, Ogden D, Ehrlich A, Hay P. Treatment for stable HIV patients in England: Can we increase efficiency and improve patient care? J Health Ser Res Policy 2014;19:131-137.

43. Stubbings T, Miller C, Humphries TL, Nelson KM, Helling DK. Telepharmacy in a health maintenance organization. Am J Health Syst Pharm 2005;62:406-410.

44. San José B, Gil MA, Puy M. Pharmaceutical care and home delivery of medication to patients with chronic myeloid leukemia. Farm Hosp 2015;39:13-22.

45. Desai KR, Chewning B, Wilcox A, Safdar N. Mail-order pharmacy experience of veterans living with AIDS/HIV. Res Social Adm Pharm 2018;14:153-161.

46. Rupp MT. Attitudes of Medicare-eligible Americans toward mail service pharmacy. J Manag Care Pharm 2013;19:564-572. 